\title{
Gender Identification Using Feature Patch-Based Bayesian Classifier
}

\author{
Shen-Ju Lin, Chung-Lin Huang, and Shih-Chung Hsu \\ Department of Electrical Engineering \\ National Tsing-Hua University, Hsin-Chu, Taiwan, R.O.C. \\ g9761531@oz.nthu.edu.tw, clhuang@ee.nthu.edu.tw, \\ chvjohnffegmail.com
}

\begin{abstract}
In the paper, we propose a Bayesian classifier which exploits nonparametric model to identify the gender from the facial images. Our major contribution is that we use feature patch-based non-parametric method to generate the posteriori of male and female based on the characteristics of the labeled training image patches. Our system consists of four modules. First, we use AAM model to identify facial feature points. Facial images are represented by the overlapping feature patches around the feature points. Second, from the labeled training patches, we select a smaller subset as the patch library based on the $\mathrm{K}$ means clustering. Third, in training, we embed the gender characteristics of the training feature patches as the posteriori of the library patches. Fourth, in testing, we integrate the posterior of the test patches to determine the gender. The experimental results demonstrate that our proposed method is better than the conventional non-feature-patch-based methods.
\end{abstract}

Keywords: Gender identification, Active Appearance Model, Patches-based Bayesian estimation.

\section{Introduction}

Biometric features of human faces reveal lots of high-level semantic information of the human such as gender, age, ethnicity and emotion expression and etc. Compared with age or ethnicity estimation, determining the gender of a facial image has become an interesting research topic. How human being identifying the gender is unknown and gender misjudgment often occurs. The facial images may contain the variation in illumination, pose, background clutter, and partial occlusion. We consider all these variations in facial image and develop a reliable method to identify the gender.

Gender identification methods can be divided into two main categories: geometrybased and appearance-based. The geometry-based category focuses on extracting the geometric feature points from facial images and describing the shape structure of the face. It uses Active Appearance Model (AAM) to build statistical model of object shape and texture information. Saatci et al. [1] propose an approach to determine the gender and expression of facial images by using AAM for feature extraction and Support Vector Machines (SVMs) for classification. Mäkinen et al. [2] present a systematic evaluation on gender classification, and show that how did face alignment influence the accuracy of gender classification methods. 
The appearance-based category can further be divided into two approaches: texture-based and statistical-based. The former uses different texture descriptors to characterize a facial image about gender, and employ machine learning algorithm to classify the gender. The texture are modeled by local binary pattern $(L B P)[3,5]$, Local Gabor binary mapping pattern (LGBMP) [4], wavelet transform [6, 7], Adaboost [8, 9], Independent Component analysis (ICA) [10]. Other researches [11] stress on the specific local region, such as noise, eyes, mouth, etc.

The statistical-based approach aims at using different features which are quantified into probability to characterize a facial image about gender according to their visual traits. Toews et al. [12] present the combination of local scale-invariant features $(S I F T)$ and object class invariant $(O C I)$ model for detecting, localizing and classifying visual traits of gender from facial images. Aghajanian et al. [13] propose a patchbased framework to determine the ambiguous within-object and replace each patch from the predefined library and frequency parameters corresponding to these patches. Li et al. [14] provide another patch-based feature representation called Spatial Gaussian Mixture Models (SGMM) to describe the image spatial information relative precisely at both local and global scales for image.

Different from [13, 14], we use non-parametric statistical method to embed the gender characteristics of the feature patches in a pre-defined patch library. In training process, we propose a Bayesian statistical framework to model the gender characteristics of the training facial images and build a posteriori gender probability distribution of the library patches. In the testing process, we integrate the gender posteriori distribution of the test image patches based on the patch library to predict the gender.

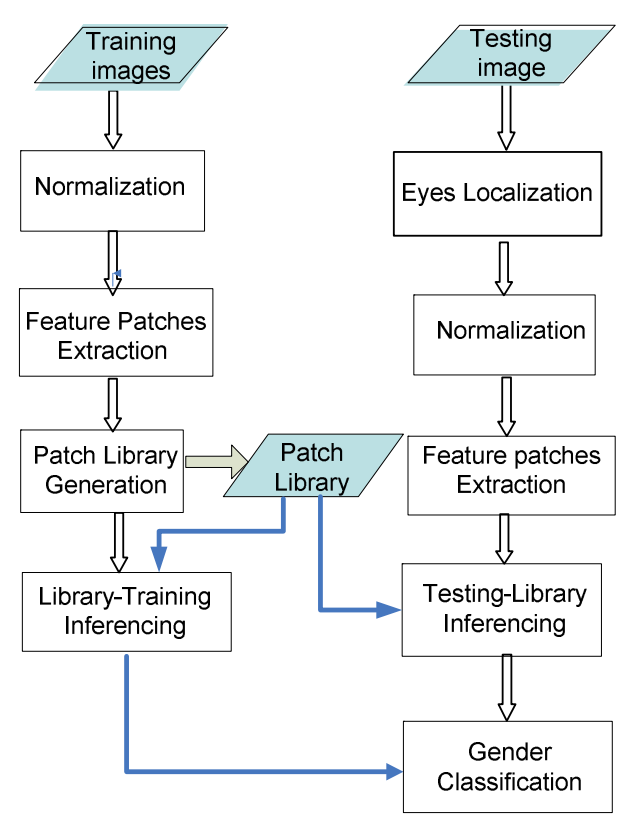

Fig. 1. The flowchart of gender estimation system 
First, we apply Adaboost algorithm [8, 9] to find the locations of eyes and then normalize the face according to the center positions of both eyes. Then, we use AAM to locate the facial feature points and then extract the overlapping feature patches of facial image. Our approach consists of four modules: (1) Select a predefined patch library from the training image patches. (2) Generate the posterior of each feature patch in the library as the off-line library-training inference. (3) Generate the posterior of each feature patch in the input test image as the on-line testing-library inference. (4) Marginalize over all the feature patches to determine the classification decision. The contributions in this paper are applying (a) multiple overlapping feature patches, (b) Bayesian gender determination framework based in the off-line library-training inference and on-line test-library inference, and (c) a library selection scheme based on eigenface with $\mathrm{K}$ means clustering.

\section{Facial Feature Points Extraction Using AAM}

Based on the MORPHY database, we may normalize and rotate the face database and apply the AAM [15] to represent the face and extract the facial feature points. Because of the different angle and size of the face, it is necessary to normalize the face image for gender estimation. Since the eyes are easily found compared to the other face features, we normalize the face images based on both eyes to adjust the orientation and the size of the face. Then we rotate the line linking two centers and scale the distance between both eyes to normalize the region size of facial features. Finally, the hair region at the top of the image is also excluded.

We apply the AAM to an input image to find the model parameters by maximizing the "match" between the model instance and the input image. Then, the model parameters are used to find the facial feature points. AAM is a well-known statistical model which consists of two parts: the shape model and the texture model. To train the AAM model, we have a set of landmark points selected as the salient points on the human face as shown in Figure 2. For each image, we use the pre-trained AAM model to search for the 27 facial feature points which are located at the corner and bag regions of both eyes, the left and right law grain regions and the hair region.

(a)

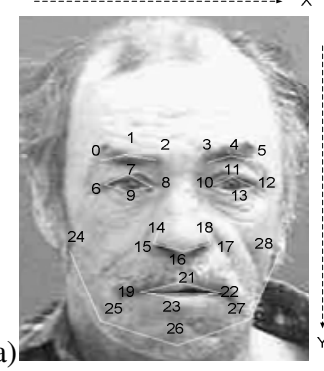

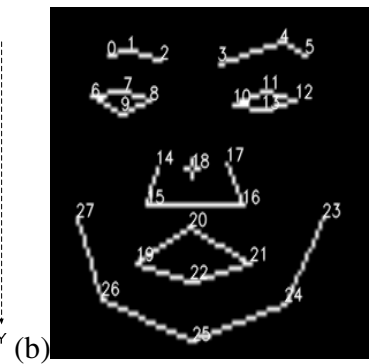

Fig. 2. (a) The label feature points of the training set. (b)The position of 28 landmark points. 


\section{Feature Patch-Based Gender Classification}

Each face image is represented by a grid of overlapping feature patches. Based on the 27 facial feature points identified by AAM, there are 27 feature patched extracted as shown in Figure 3. Each feature patch is an independent individual that provides gender information. The gender classification consists of two processes: library-totraining inference and testing-to-library inference. The first process is an off-line training process, in which we build a non-parametric statistics model to embed the training images gender characteristics into the predefined library patches. In the online gender identification process, the posteriori of each test image patches is modeled by patches from the predefined library. By integrating the posterior probability of each feature patch, we may determine the gender of the face image.
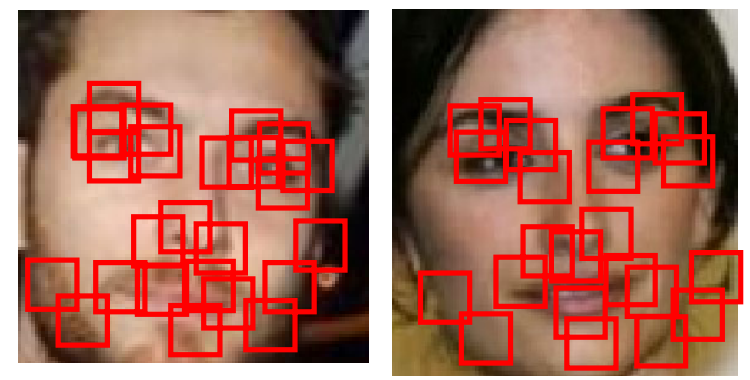

Fig. 3. The 27 selected feature patches

Gender is viewed as a class label $C$, where $C=$ Male or $\mathrm{C}=$ Female. The test image $\mathbf{Y}$ is represented as a regular grid of overlapping feature patches $\mathbf{Y}=\left[\boldsymbol{y}_{1}, \boldsymbol{y}_{2}, \ldots, \boldsymbol{y}_{N}\right]$, where $N$ is the number of selected feature patches. The resolution of the patch is $\mathrm{M} \times \mathrm{M}$. We choose a set of labeled training images from each class (male or female) to train the model. Each training image is represented by a regular grid of overlapping patches of the same size. In addition, we choose the images evenly from each of the two classes to build the predefined library, which are different from the training images. The predefined library consists of a set of overlapping feature patches which are of the same size as those in the training and testing images.

We consider the Bayesian estimation approach to identify the gender of the facial images. The idea of Bayesian estimation is that we treat each patch as a random variable and using the training images to generate a posteriori probability density of the patch. The main goal of Bayesian classification lies at the computation of the posterior probability of facial image $\mathbf{Y}, P(C \mid \mathbf{Y})$, which can be derived based on the prior $P(C)$ and the class-conditional densities $P(\mathbf{Y} \mid \mathrm{C})$. Based on the set of training set $\mathbf{X}$, we may have the Bayesian posterior probabilities $P(\mathbf{C l Y}, \mathbf{X})$. From each training image, we may extract $N(=27)$ overlapping feature patches. So, the training set $\mathbf{X}$ can be represented by $N$ patch groups as $\left\{\mathbf{X}^{p} \mid p=1, \ldots N\right\}$. Each patch group has two classes, such as $\mathbf{X}^{p}=\mathbf{X}_{\text {male }}^{p} \cup \mathbf{X}_{\text {female }}^{p}$. Here, we denote the $p$ th feature patch of the $i$ th training image as $\boldsymbol{x}_{p i} \in \boldsymbol{X}_{c}^{p}$, where $c$ indicates either male or female. 
Given the training set $\mathbf{X}$, we can obtain the posteriori probability of a facial image $\mathbf{Y}$ of class $C$ by using Bayes' rule as

$$
P(C \mid \mathbf{Y}, \mathbf{X})=P(\mathbf{Y} \mid C, \mathbf{X}) \cdot P(C) / P(\mathbf{Y}) \propto \Pi_{p=1}^{N} P\left(\boldsymbol{y}_{p} \mid C, \mathbf{X}\right) \cdot P(C)
$$

where $\boldsymbol{y}_{\mathrm{p}}$ is an individual test patch of the facial image $\boldsymbol{Y}$. Based on equation (1), we may find the posteriori $P(C \mid \mathbf{Y}, \boldsymbol{X})$, and the gender of the image $\boldsymbol{Y}$ can be determined by maximizing a posteriori as

$$
\operatorname{Gender}(\boldsymbol{Y})=\operatorname{Argmax}_{\mathrm{c}} P(C \mid \mathbf{Y}, \boldsymbol{X})
$$

Here, we have constructed the library with 27 library subsets for each class as $M_{1}^{c}, \ldots M_{27}^{c}$, of which each has $L$ feature patches as $M_{p}^{c}=\left\{\mu_{c p l} \mid l=1, \ldots L\right\} . P\left(y_{p} \mid \mu_{c p l}\right)$ with the relational information of parameter space and training set can be used to predict the gender probability of $\boldsymbol{y}_{p}$ for $p=1, \ldots . N$. To obtain the class-conditional densities $P\left(\boldsymbol{y}_{p} \mid C, \boldsymbol{X}\right)$, we do the integrating of the joint density $P\left(\boldsymbol{y}_{p}, \mu_{c p l} \mid C, \boldsymbol{X}\right)$ over a set of parameter $\left\{\mu_{p l}\right\}$ as

$$
\begin{aligned}
P\left(\boldsymbol{y}_{p} \mid C, \boldsymbol{X}\right) & =\int P\left(\boldsymbol{y}_{p}, \mu_{c p l} \mid C, \boldsymbol{X}\right) d \mu_{c p l} \\
& =\int P\left(\boldsymbol{y}_{p} \mid \mu_{c p l}\right) \cdot P\left(\mu_{c p l} \mid C, \boldsymbol{X}\right) d \mu_{c p l} .
\end{aligned}
$$

$\left\{\mu_{c p l}\right\}$ denotes a set of library patches, of which the indices $p$ and $l$ indicate the $p^{\text {th }}$ feature patch in the $l^{\text {th }}$ library image. In Eq. (3), the class-conditional densities $P\left(y_{\mathrm{p}} \mid C\right.$, $\boldsymbol{X})$ consists of a chained probability of $P\left(\boldsymbol{y}_{\mathrm{p}} \mid \mu_{c p l}\right)$ and $P\left(\mu_{c p l} l C, \boldsymbol{X}\right)$ where $P\left(\mu_{c p l} l C, \boldsymbol{X}\right)$ can be viewed as embedding the gender characteristics of the training feature patches in $\boldsymbol{X}$ into the patch library space $\left\{\mu_{c p l}\right\}$ for $c=1$ or $2, i \leq i \leq I, 1 \leq p \leq 27$, and $1 \leq l \leq L$.

For each test patch $\boldsymbol{y}_{p}$, we have two cascaded terms $P\left(\boldsymbol{y}_{p} \mid \mu_{c p l}\right)$ and $P\left(\mu_{p l} \mid C, \boldsymbol{X}\right)$ which are modeled by two inference processes: testing-library inference and library-training inference. To determine the gender of the input image, we (1) cascade the two inferences, (2) integrate all patches, (3) multiple the gender likelihood of all the patches of the test image as $\Pi_{p=1}^{N} P\left(\boldsymbol{y}_{p} \mid C, \boldsymbol{X}\right)$, and (4) find the maximum posterior of $P(C \mid \boldsymbol{Y}$, $\boldsymbol{X})$ to determine the gender.

\subsection{Patch Similarity Measure}

Each pixel in the feature patch can be described by a set of local binary pattern ( $L B P$ ) codes $[3,5]$. $L B P$ code encodes the local structure around each pixel in the feature patch. The histogram of $L B P$ labels calculated over the patches can be exploited as a texture descriptor. A popular measure between two feature patches in terms of two normalized histogram of $L B P$ labels $p(u)$ and $q(u)$ is the Bhattacharyya coefficient. For discrete densities such as the normalized $L B P$ histograms $p(u)=\left\{p^{(u)}\right\}_{u=1 \ldots \mathrm{m}}$ and $q(u)=\left\{q^{(u)}\right\}_{u=1 \ldots \mathrm{m}}$, the correlation coefficient is defined as

$$
\rho[p, q]=\sum_{u=1}^{m} \sqrt{p^{(u)} q^{(u)}}
$$

The larger $\rho$ is, the more similar these two feature patches are. The similarity between two patches can be defined as

$$
d=\sqrt{1-\rho[p, q]} .
$$


Dissimilar distributions result in a larger $d$. Furthermore, the likelihood can be written as

$$
p\left(x \mid \mu_{l}\right)=\frac{1}{\sqrt{2 \pi} \sigma} e^{-\frac{d^{2}}{2 \sigma^{2}}}
$$

The likelihood is specified by a Gaussian with variance $\sigma$. So, we may have probability density distribution as

$$
P\left(\boldsymbol{x} \mid \mu_{c p l}\right)=P\left(\boldsymbol{x} \mid \mu_{c p l}\right) / \Sigma_{l=l}^{L} P\left(x \mid \mu_{c p l}\right)
$$

where $P\left(\boldsymbol{x} \mid \mu_{c p l}\right)$ is a probability density distribution with $L$ bins for $\mu_{c p 1}, \mu_{c p 2}, \ldots \mu_{c p L}$.

\subsection{Training Process}

The training set $\boldsymbol{X}$ consists of two labeled training images sets as $\boldsymbol{X}=\mathbf{X}_{\text {male }} \cup \mathbf{X}_{\text {female }}$ which are further decomposed into $N$ labeled patch groups as $\boldsymbol{X}_{\text {male }}=$ $\left\{\boldsymbol{X}_{\text {male }}^{p} \mid p=1, \ldots N\right\}$ and $\boldsymbol{X}_{\text {female }}=\left\{\boldsymbol{X}_{\text {female }}^{p} \mid p=1, \ldots N\right\}$. We may also describe the two labeled training sets as $\mathbf{X}_{\text {male }}=\left\{\boldsymbol{x}_{c p i} \mid C=1, p=1, \ldots N, i=1, \ldots I\right\}$ and $\mathbf{X}_{\text {female }}=\left\{\boldsymbol{x}_{\text {cpi }} \mid C=2\right.$, $p=1, \ldots N, i=1, \ldots I\}$. The library can be further divided into subgroup $M_{p}^{c}$, where the indices $c$ and $p$ indicate the $p^{\text {th }}$ subset of class $C$.

Before calculate $P\left(\mu_{c p l} \mid C, \boldsymbol{X}\right)$, we use Bayesian rule to derive the posteriori density $P\left(\mu_{c p l} \mid C, \boldsymbol{x}_{c p i}\right)$ over the parameters space $\left\{\mu_{c p l}\right\}$. We simplify $P\left(\mu_{c p l} \mid C, \boldsymbol{x}_{c p i}\right)=$ $P\left(\mu_{c p l} \mid \boldsymbol{x}_{c p i}\right)$ for specific class $c$ which is defined as

$$
P\left(\mu_{c p l} \mid x_{c p i}\right)=\frac{P\left(x_{c p i} \mid \mu_{c p l}\right) P\left(\mu_{c p l}\right)}{P\left(x_{c p i}\right)}
$$

where $\boldsymbol{x}_{c p i}$ denotes the $p^{\text {th }}$ feature patch in the $i^{\text {th }}$ training image of specific class $c$. The prior $P\left(\mu_{c p l}\right)$ indicates the weight of the $l$ th patch in the library subset $M_{p}^{c}$ which is determined by the frequency of the designated patches being selected in the training process. $P\left(\mu_{c p l}\right)$ is normalized by $P\left(\mu_{c p l}\right) / \Sigma_{l} P\left(\mu_{c p l}\right)$. Then, we have

$$
P\left(\mu_{c p l} \mid C=c, \boldsymbol{X}\right)=\int \mathrm{P}\left(\mu_{c p l} \mid C, \boldsymbol{x}_{c p i}\right) d \boldsymbol{x}_{c p i} / \Sigma_{l} \mathrm{P}\left(\mu_{c p l} \mid C, \boldsymbol{X}\right)
$$

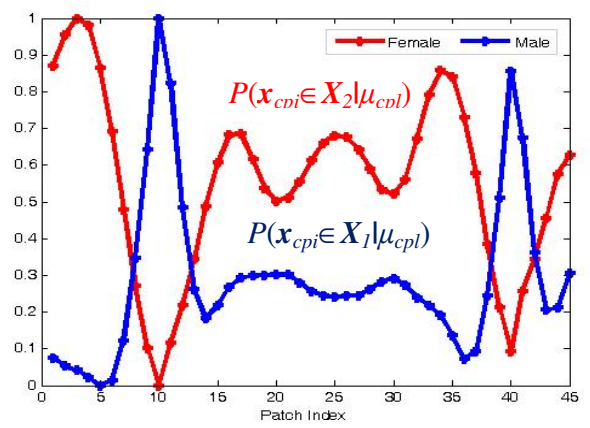

Fig. 4. The likelihood density functions $P\left(\boldsymbol{x}_{c p i} \in \boldsymbol{X}_{l} \mid \mu_{c p l}\right)$ and $P\left(\boldsymbol{x}_{c p i} \in \boldsymbol{X}_{2} \mid \mu_{c p l}\right)$ for every library patch $\mu_{c p l}$, where $\mathbf{X}_{1}=\mathbf{X}_{\text {male }}$ and $\mathbf{X}_{2}=\mathbf{X}_{\text {female }}$. 
To find the posteriori density $P\left(\mu_{c p l} l, \boldsymbol{X}\right)$ for class $c$, we calculate the likelihood term $P\left(\boldsymbol{x}_{c p i} \mid \mu_{c p l}\right)$ of the labeled training sets $\mathbf{X}_{\text {male }}$ and $\mathbf{X}_{\text {female }}$ over the pertinent parameters $\mu_{c p l}$, and exploit the relationship of the corresponding feature patch similarity between $\boldsymbol{x}_{c p i}$ and $\mu_{c p l}$. For each $\mu_{c p l}$, we have the likelihood function $P\left(\boldsymbol{x}_{c p i} \mid \mu_{c p l}\right)$ for each library patch $\mu_{c p l}$ as shown in Figure 4.

We use the maximum a posteriori estimator $(M A P)$ to find the highest similarity between the training feature patch $\boldsymbol{x}_{c p i}$ and all the possible library feature patch $\mu_{c p l}$ in $M_{p}^{c}$. We search all $\mu_{c p l}$ in $M_{p}^{c}$ for the highest similar one with the training feature patch. For every training feature path $\boldsymbol{x}_{c p i}$, we find the most similar library feature patch in $M_{p}^{c}$ as

$$
\mu_{c p l *}=\operatorname{Argmin}_{l} \operatorname{Dis}\left(\mu_{c p l}, \boldsymbol{x}_{c p i}\right) \text { for each } \boldsymbol{x}_{c p i}
$$

where $\mu_{c p l} \in M_{p}^{c}$, and $M_{p}^{c}$ is the $p$ th library subset, and $D i s\left(\mu_{c p l}, \boldsymbol{x}_{c p i}\right)$ is calculated by using eq. (4).

To find the likelihood function $P\left(\boldsymbol{x}_{c p i} \mid \mu_{c p l}\right)$ for specific library patch, we search all training feature patches to find the similarity. Considering $I$ independent training feature patches $\boldsymbol{x}_{c p i}, i=1, \ldots I$, we can represent the likelihood of the training patches, $P\left(\boldsymbol{x}_{c p i} \mid \mu_{c p l}\right)$, related to the specific parameter $\mu_{c p l}$. With $P\left(\boldsymbol{x}_{c p i} \mid \mu_{c p l}\right)$, we may obtain the posteriori distribution for the parameter set $\left\{\mu_{c p l}\right\}$ as

$$
P\left(\mu_{c p l} \mid \boldsymbol{x}_{c p i}\right) \propto P\left(\boldsymbol{x}_{c p i} \mid \mu_{c p l}\right) \cdot P\left(\mu_{c p l}\right) / P\left(\boldsymbol{x}_{c p i}\right)
$$

where $P\left(\mu_{c p l}\right)$ is the prior of the parameter $\mu_{c p l}$. The training feature patches correlated with predefined library feature patch are used to construct the posterior $P\left(\mu_{c p l} \mid \boldsymbol{x}_{c p i}\right)$.

\subsection{Testing Process}

To predict the probability of the class of the input feature patch $\boldsymbol{y}_{p}$, we need to compute the likelihood $P\left(\boldsymbol{y}_{p} \mid \mu_{c \mathbf{p}}\right)$ by exploiting the feature patch similarity between $\boldsymbol{y}_{\mathrm{p}}$ and $\mu_{c \mathbf{p}}$. Then, we may combine the probability $P\left(\boldsymbol{y}_{p} \mid \mu_{c p l}\right)$ and $P\left(\mu_{c p l} \mid \boldsymbol{X}\right)$ to obtain the joint conditional density, $P\left(\boldsymbol{y}_{p}, \mu_{c p l} \mid C=c, \boldsymbol{X}\right)$. For each feature patch $\boldsymbol{y}_{p}$, we may find its similarity with the library feature patch $\mu_{c p l}$ described as

$$
p\left(\mu_{c p l} \mid y_{p}\right)=\frac{1}{\sqrt{2 \pi} \sigma} e^{-\frac{d^{2}}{2 \sigma^{2}}}
$$

where $d$ is the similarity measure between two feature patches (Equ. (5)). We use the Bayes' rule to calculate the posteriori density $P\left(y_{p} \mid \mu_{c p l}\right)$ over the parameters $\left\{\mu_{c p l}\right\}$ as

$$
P\left(y_{p} \mid \mu_{c p l}\right)=\frac{P\left(\mu_{c p l} \mid y_{p}\right) P\left(y_{p}\right)}{P\left(\mu_{c p l}\right)}
$$

where prior $P\left(y_{p}\right)$ indicates the weight of the $p t h$ test patch which is determined by the frequency of the designated patches being selected in the testing-library inference process.

To find the posteriori density $P\left(\boldsymbol{y}_{p} \mid \mu_{c p l}\right)$, we calculate the likelihood term $P\left(\mu_{c p l} \boldsymbol{y}_{p}\right)$ based on the subset $M_{p}^{c}$ by exploiting the patches similarity measure (eq. (12)). We use the maximum a posteriori estimator (MAP) to find the highest similar feature 
patch $\boldsymbol{y}_{p}$ for all the possible library feature patch $\mu_{c p l}$ in $M_{p}^{c}$. For every $\mu_{c p l}$, we find $\boldsymbol{y}_{p}$ selected if

$$
\operatorname{Dis}\left(\boldsymbol{y}_{p}, \mu_{c p l}\right)>\theta_{p}
$$

where $\operatorname{Dis}\left(\boldsymbol{y}_{p}, \mu_{c p l}\right)$ is calculated by using eq. (4), and $\theta_{p}$ is the similarity threshold. For each $\mu_{c p l}$, we use (14) to determine whether the specific $\boldsymbol{y}_{p}$ is selected or not, and then accumulate the number of each $\boldsymbol{y}_{p}$ being selected for all $\mu_{c p l}$. We divide the number by the total accumulated number of the all $\boldsymbol{y}_{p}$ as the priori $P\left(\boldsymbol{y}_{p}\right)$, so that $\Sigma_{p} P\left(\boldsymbol{y}_{p}\right)=1$. With likelihood term $P\left(\mu_{c p l} \mid y_{p}\right)$ and $P\left(y_{p}\right)$, we may have the posteriori density $P\left(y_{p} \mid \mu_{c p l}\right)$.

We construct the male and female posteriori distribution by using on the male and female training images. During the identification process, for every test patch, we generate its posterior information by search all library patches based on their similarity. Finally, we can integrate overall those patches from the facial image to provide a posterior probability and then determine the gender.

The male and female accumulated distribution for the same library image is obviously different, the extent of accumulated distribution corresponding to the gender can illustrate that the possibility of the local region in the library image belongs to the specific gender. The difference of the male and female specific accumulated distribution can represent the corresponding specific region in the library image having discriminating ability about gender.

\section{$4 \quad$ Library Selection Scheme}

The formation of predefined library is a crucial step for gender identification. In the training process, we calculate the accumulated frequency of all possible patches within the predefined library. In the inference process, we exploit the accumulated information of library patch which contain the highest similarity with the corresponding test patch. Basically, the library feature patches are obtained from the facial images of various kinds of changes such as in illumination, pose, and background clutter. We find the relation of the variation of predefined library composition with regard to the classification accuracy.

We propose a method by using the characteristic of eigenface with K-mean clustering for library selection. The eigenface method is based on principle component analysis $(P C A)$, used to find a suitable low-dimensional space. There are two primacy procedures: (a) Eigenspace generation. Given $I$ normalized facial image patches for training, and each feature patch is represented as $\mathrm{M} \times \mathrm{M}$. These feature patches are converted into the column vector type with the dimension $\mathrm{M}^{2} \times 1$. (b) Projection onto eigenspace. Each of the training image patch $\boldsymbol{x}_{i}$ is projected onto the eigenspace as the weighting vectors. Weighting vector is calculated by the eigenspace and the average patch vector with inner product. The weighting vectors are considered as the feature points in $K$-dimension space.

With the weighting vectors from these patches, we execute the cluster analysis to group the weighting vectors by the variety of characteristics. Here, we choose $K$ means clustering to partition $I$ patches into $k$ clusters in which each patch is associated one of the clusters with the nearest mean, and the weighting vectors is viewed as the observations. After $K$-mean clustering, we choose each cluster centroid and the fea- 
ture point with the minimum Euclidean distance as the selected patch. Finally, we collect these selected patches as a library.

\section{Experimental Results}

Here, we evaluate our gender classification algorithm by using different formation of library images. As shown in Figures 5.1 5.3, our face images are obtained from: (1) Wild database contains more than 13000 face images collected from 5749 people. (2) Caltech 10,000 web faces database contains 10524 human faces with various resolution and settings. (3) Color FERET database. In our experiment, we only use the fa partition in FERET database, i.e., the regular frontal facial images. It contains 1364 images.

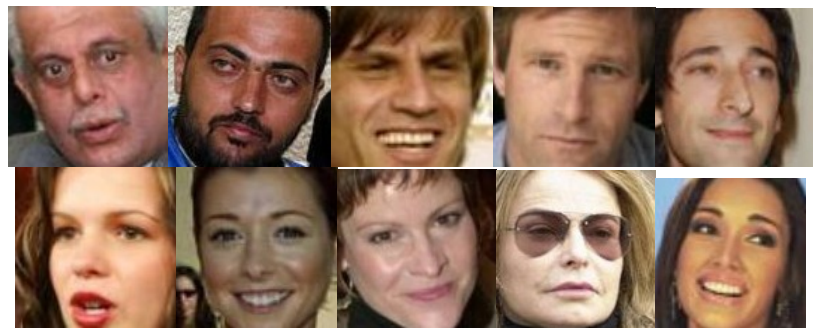

Fig. 5.1. Five male/female facial image in Labeled Faces in the Wild database

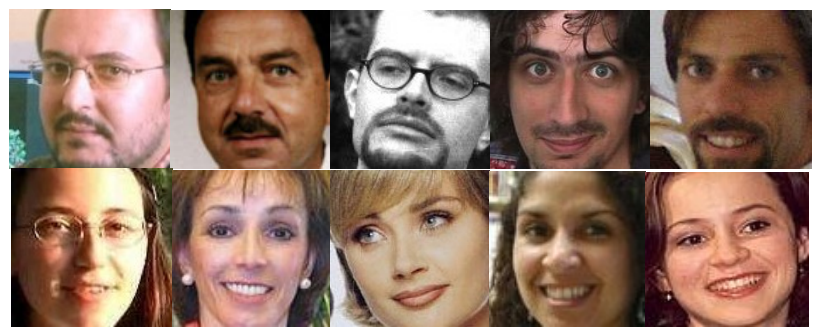

Fig. 5.2. Five male/female facial image in Caltech 10,000 web faces database
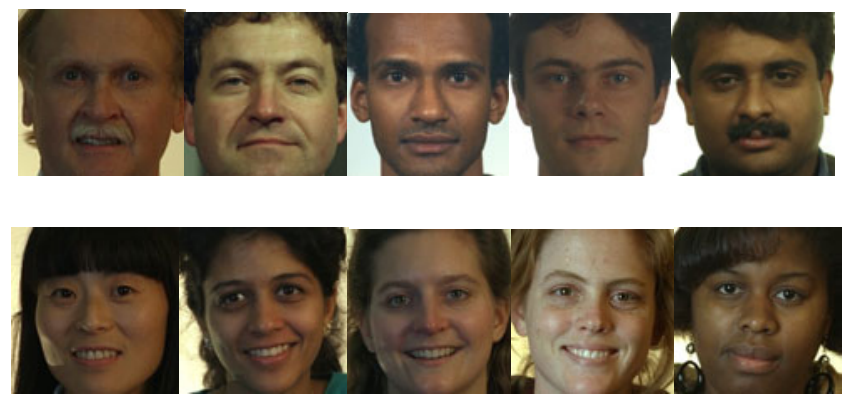

Fig. 5.3. Five male/female facial images in Color FERET database 
From the male and female image patches, we select some patches as the library and let the remaining as the training image patches. We illustrate the difference of accurate gender identification according to the different library formation. To describe the patch information precisely, we set the size of patch to $6 \times 6$ grid block uniformly. Then, we select the testing set which consist 500 male and female images with various kinds of variations (lighting, expression, pose, background...etc). In the following, we show four different experiments and compare the performance of our method with the non-featurepatch-based method of which the library images are selected randomly.

(1) Experiment 1. The training set consists of 8000 male and 8000 female images to build the model. Then, we regulate the test images with two different resolution $(30 \times 30,60 \times 60)$ and 7 different library formations $(L=2,10,30,60,120,240,360)$ to show the difference of correct gender prediction. We compare the recognition rate of our method with [13] as illustrated in Figure 6 to show that our method are better than the random selection method in most cases. Our method uses more discriminative male and female patches so that the accuracy rate of our method is better. Smaller $L$ indicates less information in the library, and the detection accuracy rate is lower. With more library patches, we have higher detection rate. By using the discriminative library patches with variation, our method shows better performance.
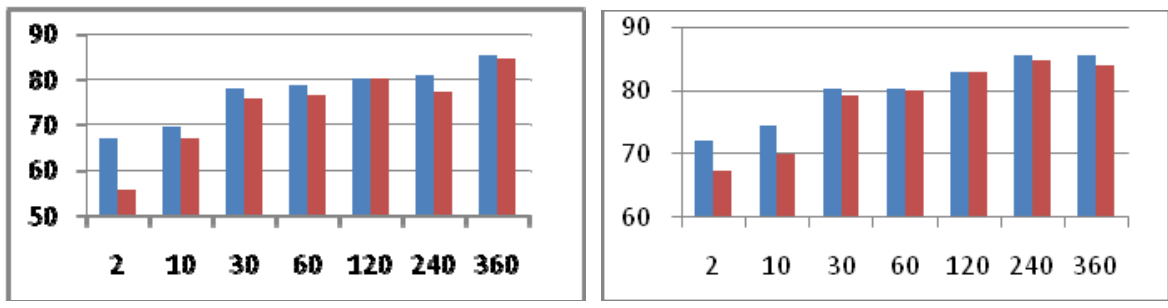

Fig. 6. The accuracy comparison of our method and [13] using (a) $30 \times 30$ test images. (b) $60 \times 60$ test images. (Training set: 8000 male and female images).

(2) Experiment 2. The smaller training set consists of 4000 male and 4000 female images to build the model, and select 7 different library formations $(L=2,10,30,60$, $120,240,360)$. We also let the test images with two different resolution $(30 \times 30$, $60 \times 60)$ and compare the gender identification rates of our method with [13] as illustrated in Figure 7. The experimental results show that the selection of library and training images from the smaller image database can still maintain a good gender classification performance. However, smaller $L$ indicates less information in the library, and the detection rate is lower. Figure 7(b) shows that, for $L=120$, gender classification accuracy of our method and [13] are $85.5 \%$ and $82.5 \%$, respectively.

(3) Experiment 3. We use the fa partition from color FERET database to verify our gender identification algorithm. The frontal facial images consist of 861 males and 503 females. Experiment 2 demonstrates that using clustering library selection with 240 library patches with the resolution $60 \times 60$ retains the best accurate gender identification rate. Using library with lots of image patches will provide more gender accumulated distribution information. The library does not contain enough characteristics of FERET 

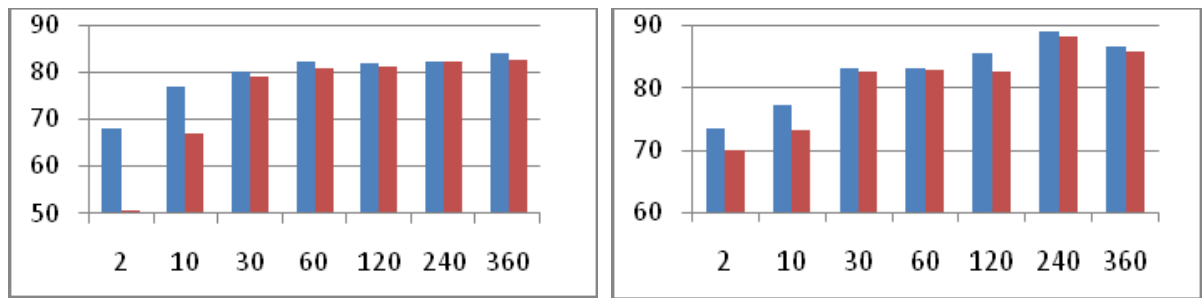

Fig. 7. The accuracy comparison of our method and [13] using (a) $30 \times 30$ test images. (b) 60×60 test images. (Training set: 4000 male and female images).

frontal facial images, so that, we have a less accurate gender prediction for FERET frontal facial images. The recognition accuracy of our method is $78.01 \%$ compared with $76.39 \%$ of the method [13].

(4) Experiment 4. We use Bao face database images for gender classification experiment from and show some gender detection results in Figures 8. We use two different library selection methods with $L=240$, and each face image with the resolution $60 \times 60$, respectively. Based on the experimental results shown in Figure 8, we find that our method is better than [13]. The experimental results of different library formations are summarized and shown in Tables 1 and 2.

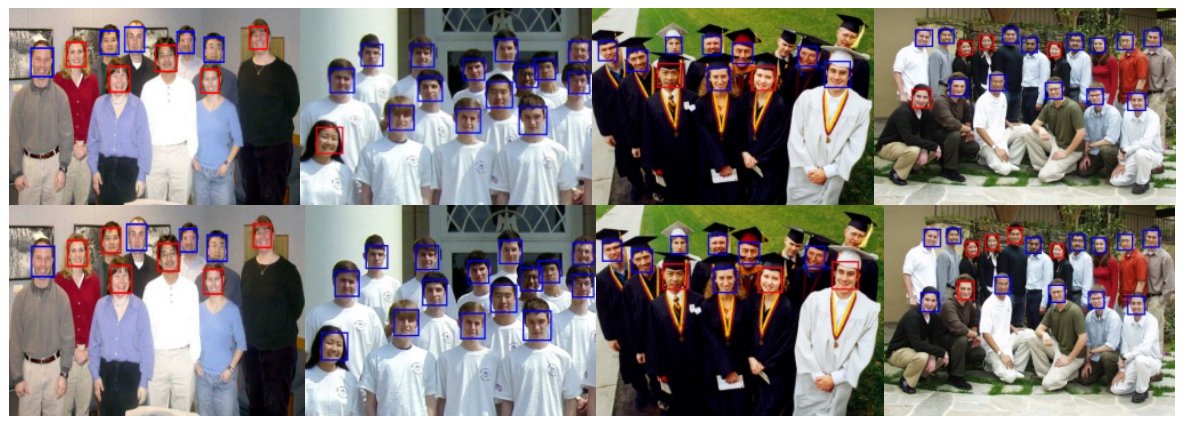

Fig. 8. Some gender detection result of the images use by the model with eigenface with clustering selection (Top) and random selection (Bottom)

Table 1. The recognition rate of two methods with different library $L=240 / L=120$ with resolution $60 \times 60$

\begin{tabular}{|c|c|c|}
\hline Recognition rate & Our method & Random selection[13] \\
\hline $\mathrm{L}=240$ & $88.7 \%$ & $86.8 \%$ \\
\hline $\mathrm{L}=120$ & $85.5 \%$ & $82.5 \%$ \\
\hline
\end{tabular}

Table 2. The accuracy comparison with library $L=8 / L=20$, and image resolution $60 \times 60$

\begin{tabular}{|c|c|c|}
\hline Recognition rate & Our method & Random selection[13] \\
\hline $\mathrm{L}=8$ & $83.4 \%$ & $80.03 \%$ \\
\hline $\mathrm{L}=20$ & $72.9 \%$ & $72.1 \%$ \\
\hline
\end{tabular}




\section{Conclusions}

We have proposed a modified Bayesian estimation framework to exploit patch similarity and accumulated distribution to predict human gender of the facial images. We propose a library selection scheme to choose the discriminative male and female images based on $\mathrm{K}$ means clustering, and build the male and female accumulated distribution based on the characteristics of the labeled training images. The experimental results demonstrate that our proposed method is better than the conventional method.

\section{References}

[1] Saatci, Y., Town, C.: Cascaded Classification of Gender and Facial Expression Using Active Appearance Models. In: AFGR 2006, pp. 393-400 (April 2006)

[2] Mäkinen, E., Raisamo, R.: Evaluation of Gender Classification Methods with Automatically Detected and Aligned Faces. IEEE Trans. on PAMI 30(3), 541-547 (2008)

[3] Lian, H.C., Lu, B.L.: Multi-View Gender Classification Using Multi-Resolution Local Binary Patterns and Support Vector Machines. Int. J. of Neural Systems 17(6) (2007)

[4] Xia, B., Sun, H., Lu, B.L.: Multi-View Gender Classification Based on Local Gabor Binary Mapping Pattern and Support Vector Machines. In: IEEE ICNN, p. 3388 (2008)

[5] Fang, Y., Wang, Z.: Improving LBP Features For Gender Classification. In: Int. Conf. on Wavelet Analysis and Pattern Recognition, pp. 373-377 (August 2008)

[6] Li, J., Lu, B.-L.: A Framework for Multi-view Gender Classification. In: Ishikawa, M., Doya, K., Miyamoto, H., Yamakawa, T. (eds.) ICONIP 2007, Part I. LNCS, vol. 4984, pp. 973-982. Springer, Heidelberg (2008)

[7] Leng, X.M., Wang, Y.D.: Gender Classification Based on Fuzzy SVM. In: Int. Conf. on Machine Learning and Cybernetics (2008)

[8] Baluja, S., et al.: Boosting Sex Identification Performance. Int. J. of Computer Vision 71(1), 111-119 (2007)

[9] Shen, B.-C., Chen, C.S., Hsu, H.H.: Fast Gender Recognition by Using a Shared Integral Image Approach. In: IEEE ICASSP (2009)

[10] Wang, Z.-H., Mu, Z.-C.: Gender Classification Using Selected Independent-Features Based on Genetic Algorithm. In: 8th Int. Conf. on Machine Learning and Cybernetics, pp. 394-398 (July 2009)

[11] Andreu, Y., Mollineda, R.A.: On the Complementarity of Face Parts for Gender Recognition. In: Ruiz-Shulcloper, J., Kropatsch, W.G. (eds.) CIARP 2008. LNCS, vol. 5197, pp. 252-260. Springer, Heidelberg (2008)

[12] Toews, M., Arbel, T.: Detection, Localization, and Sex Classification of Faces from Arbitrary Viewpoints and under Occlusion. IEEE Trans. on PAMI 31(9) (September 2009)

[13] Aghajanian, J., et al.: Within-Object Classification. In: IEEE 12th ICCV (2009)

[14] Li, Z., Zhou, X., Huang, T.S.: Spatial Gaussian Mixture Model for Gender Recognition. In: IEEE ICIP (November 2009)

[15] Cootes, T.F., Wheeler, G.V.: View-Based Active Appearance Models. Image and Vision Computing 20, 657-664 (2002) 